\title{
Utilização de serviços de saúde de atenção básica em municípios da metade sul do Rio Grande do Sul: análise baseada em sistemas de informação
}

\author{
Use of primary health care services in municipalities \\ in the southern half of the Rio Grande do Sul State: \\ analysis based on information systems
}

\author{
Tatiana Engel Gerhardt ${ }^{1}$ \\ Juliana M aciel Pinto ${ }^{1}$ \\ Deise Lisboa Riquinho ${ }^{1}$ \\ Adriana Roese ${ }^{2}$ \\ Daniel Labernarde dos Santos ${ }^{1}$ \\ M aristela Correa Rodrigues de Lima ${ }^{1}$
}

${ }^{1}$ Escola de Enfermagem, Universidade Federal do Rio Grandedo Sul. RuaSão Manoel 963, Santana. 90620-110 Porto Alegre RS. tatiana.gerhardt@ufrgs.br ${ }^{2}$ UniversidadeFederal de Pelotas.
Abstract This study is proposed to describe the supply and demand of Primary Health CareService in thirteen municipalities of the southern half of Rio Grande do Sul State. For analysis, secondary data were obtained from the Data Processing D epartment of the U nified Health System (DATASUS). Data from appointments, procedures and structure of Primary Health Care Service were associated with concepts present in public policies and specific legal authorizations. A quantitative study was carried out through the analyses of absolute frequencies of appointments and procedures from 2000 to 2005. Results showed diversified situations requiring primary health care service, which allowed different interpretations for the situations found. Among them, it can be cited the existence of a database with subnotifications of appointments and procedures or even theabsence of demand and/or supply for those within the municipalities researched. It is suggested the improvement of the mechanisms available for the analysis of primary health care services, which will enable the outlining of possible workflows, trajectories and therapeutic itineraries of users as well as the unveiling of likely local disparities regarding health.

Key words Primary health care, Health planning, Information systems, $\mathrm{H}$ ealth services/utilization
Resumo Esteestudo propõe se a descrever a oferta e demanda de serviços de saúde de atenção básica em treze municípios da metade sul do Estado do Rio Grande do Sul, por meio de dados secundários provenientes do site do Departamento de Informática do Sistema Ú nico de Saúde (DATASUS). Foram associados dados de consultas, deprocedimentos e de estrutura em serviços de saúde na atenção básica a conceitos presentes em políticas públicas e portaria específica. Realizou-se um estudo quantitativo, analisando frequências absolutas de consultas e procedimentos no período de 2000 a 2005. Os resultados mostram uma diversidadedesituaçõesna atenção básica em saúde, possibilitando diferentes interpretações para as situações encontradas. Entreelas, pode-se citar a existência de bancos de dados com subnotificações de consultas e procedimentos, ou mesmo a não oferta e/ou demanda por tais atendimentos. Sugerese 0 aprimoramento dos mecanismos disponíveis para a análise dos serviços de saúde de atenção básica, o que facilitará o delineamento dos possíveis fluxos, trajetórias e itinerários terapêuticos dos usuários e o desvelamento de possíveis disparidades locais em saúde.

Palavras-chave Atenção primária à saúde, Planejamento em saúde, Sistemas de informação, Serviços de saúde/utilização 


\section{Introdução}

Este trabalho busca analisar as relações existentes na oferta edemanda deserviços desaúdeem treze municípios da metade sul do Rio Grande do Sul, identificando as formas de utilização dos serviços de saúde de atenção básica $(A B)$ nos municípios estudados. É parte do projeto intitulado "Fluxos eutilização de serviços de saúde: mobilidadeenecessidades em saúde de usuários enovos desafios para a integralidade em saúde pública", que tem como objetivo principal estudar a temática defluxos eutilização deserviços de $A B$ e de média complexidade (MC) em algunsmunicípios da metade sul do Rio Grande do Sul, a partir da oferta e da demanda por esses serviços ${ }^{1}$.

De certa forma, os dados aqui analisados envolvem a discussão de princípios e diretrizes do SistemaÚ nico de Saúde (universalidade, equidade, integralidade e organização da rede regionalizada e hierarquizada) e a forma como são apropriados e efetivados no âmbito da gestão e do planejamento em saúde². As diretrizes organizacionais (descentralização, hierarquização e regionalização dos serviços de saúde) constituem a base operacional para queo sistema de saúde efetive os princípios propostos (universalidade, equidadee integralidade).

A efetiva implantação do Sistema Único de Saúde (SUS) tem mostrado a necessidade de mudanças nos órgãos gestores para superar as ineficiências da assistência à saúde prestada à população. Durante o processo demunicipalização, muitos dos princípios e diretrizes constitucionais, que garantem o direito à saúde, não se transformaram em ações concretas suficientes para a efetiva implementação de mudanças. A conformação de um novo modelo assistencial, baseado na organização da rede de serviços regionalizada e hierarquizada e de ações sob gestão municipal, demanda a existência de sistemas de informação em saúde (SIS) capazes de alimentar o planejamento e a gestão locais.

O planejamento dependedediagnósticos precisos e sensíveis para a elaboração de objetivos e metas para a própria prática avaliativa. Bons diagnósticos exigem boas bases de informação. Os SIS revelam-se instrumentos imprescindíveis e extremamente úteis no cumprimento das atribuições conferidas à direção municipal do SUS pela Lei Orgânica da Saúden ${ }^{\circ}$ 8.080/903: planejar, organizar, controlar e avaliar as ações e os serviços de saúde e gerir e executar os serviços públicos de saúde". Existem diversos elementos passíveis de avaliação, que vão desde as informações de caráter administrativo (recursoshumanos, materiais, etc.), a situação de saúde (perfil epidemiológico e sanitário), e também a utilização de serviços (oferta e demanda de serviços de saúde).

A oferta de serviços de saúde depende da capacidade instalada do serviço, dos recursos humanos disponíveis e das condições para o acesso geográfico e funcional ao serviço. Enquanto que "as demandas por serviços de saúde resultam da conjugação de fatores sociais, individuais e culturais prevalentes na população" ${ }^{4}$. Assim, iniquidades na oferta de serviços, ou mesmo em fatores que influenciam na sua demanda, acabam por delinear o perfil de utilização dos serviços.

A análise das ofertas e necessidades de saúde da população potencializa os trabalhadores, a equipe, os serviços e a rede a pautar suas intervenções e práticas dentro do que as pessoas buscam como cuidado à sua saúde, visando a uma atenção mais humanizada e qualificada, respondendo ao indivíduo e à coletividade em suas ansiedades e necessidades em saúde ${ }^{5}$.

Uma estratégia para identificar o perfil de uso de serviços de saúde está na utilização de dados secundários organizados pelos SIS. Esses dados, sejam eles coletados semanal, diária, mensal ou anualmente, são importantes ferramentas para elaboração de um plano inicial de ação em saúde, guiando gestores e profissionais na priorização de ações a serem implementadas. Para tanto, o aprimoramento dos SIS e a adequação dos profissionais ao seu uso mostram-se cada vez mais importantes para o planejamento em saúde.

Estudar a oferta e a demanda por serviços de saúde (consultas e procedimentos) a partir de dados secundários pressupõe uma série de desafios, seja pela variedade de fontes de dados existentes, seja pela busca que deve ser feita para encontrar esses dados. Essa abordagem permite a visualização das características estruturais e organizacionais de um serviço de saúde em um período determinado, sem que haja a necessidade de recorrer ao local onde o serviço é prestado.

Acredita-se que, por meio da coleta e análise dos dados secundários, associados a documentos disponíveis para organizá-los e avaliá-los, pode-se gerar informações pertinentes ao planejamento em saúde na região em estudo. Desta maneira, pretende-se favorecer a reflexão acerca da organização dos serviços locais de saúde esua utilização, vistos a partir da ótica dos SIS e das políticas públicas existentes para a regulação da rede de serviços em saúde, possibilitando a identificação de estratégias para o aprimoramento dessas políticas. 


\section{Métodos}

Esteéum estudo descritivo e exploratório, realizado através de método quantitativo. A análise dos dados coletados foi univariada e frequencial, realizada por meio do software Excel.

A população em estudo envolveu trezemunicípios da metadesul do Rio Grande do Sul sendo eles Amaral Ferrador, Arambaré, Camaquã, Canguçu, Cerro Grande do Sul, Chuvisca, Cristal, Dom Feliciano, Encruzilhada do Sul, Santana da Boa Vista, São Lourenço do Sul, Sentinela do Sul e Tapes. Referentes a esses municípios, foram utilizados dados secundários provenientes do Sistema de Informações Ambulatoriais do SUS (SIA/DATASUS), para os anos de 2000 a 2005, e do Cadastro Nacional de Estabelecimentos de Saúde (CNES/DATASUS), todos coletados no primeiro semestre de 2006. Além desses dados, foram utilizadas estatísticas populacionais do Instituto Brasileiro de Geografia e Estatística (IBGE) para o mesmo período, coletadas indiretamente através do site do DATASUS, em 2006.

Utilizou-seo SIA/DATASU S como instrumento de coleta dos dados, pois é um dos principais sistemas de interesse à saúde no Brasil. Criado nacionalmenteem 1991 e com a mesma lógica do Sistema de Informações Hospitalares do SUS (SIH/DATASUS), ou seja, a apuração de custose o pagamento de serviços realizados, substituindo os sistemas da previdência social ${ }^{6}$, esse sistema conta com o Boletim de Produção Ambulatorial (BPA), onde são registradas as informações a respeito do procedimento ambulatorial realizado. Desta forma, as secretarias de saúde podem conhecer e acompanhar os serviços produzidos em suas unidades.

Por ser um documento voltado para os recursos financeiros, há al gumas dificuldades para utilizar os dados coletados para o planejamento em saúde no que refereà $A B$, uma vez que a maioria das ações da $A B$ em saúde tem um financiamento fixo per capita, ou em pacotes conforme os programas. M esmo assim, o SI A/DATASUS é o sistema responsável pela captação e pelo processamento das contas ambulatoriais do SUS, totalizando um montante de mais de $130 \mathrm{mi}$ Ihões de atendimentos por mês?

0 grupo de procedimentos contidos no banco de dados do SI A/DATASU S escol hido para este estudo foi o 07, que descreve os procedimentos realizados por profissionais médicos e outros de nível superior e médio. O ptou-se por este grupo por conter dados referentes a procedimentos realizados por diferentes profissionais da saúde, sabendo-se que a equipe multidisciplinar é condição indispensável ao atendimento na $A B$ em saúde no Brasil e que se constitui minimamente por profissionais médicos e de enfermagem.

Os dados escol hidos, coletados e armazenados em arquivos no software Excel, foram organizados conforme o conjunto de consultas e procedimentos da $A B$ ampliada ${ }^{8}$ preconizada pela Norma Operacional da Assistência à Saúde (NOAS-SUS) e por município. Os procedimentos básicos, as consultas básicas não urgentes e as consultas básicas de urgência da $A B$ ampliada foram dispostos em tabela única, a qual também foram acrescentados os parâmetros correspondentes ao tipo de consulta. Importante ressaltar que não há parâmetros avaliativos para todos os diferentes tipos de consultas na $A B$ etambém nenhuma forma de avaliar a quantidade e variedade apresentada em procedimentos básicos.

A NOAS-SUS prevê as responsabilidades em ações e serviços de saúde para as três esferas governamentais e define a tabela de procedimentos econsultas a serem executados no âmbito da $A B$. A partir da NOAS-SUS, a idéia de construção de um Plano Diretor de Regionalização da Saúde (PDR) que servisse como instrumento de ordenamento do processo de regionalização da assistência à saú deem cada estado brasileiro foi efetivada. Para tanto, no presente estudo, foram utilizados conceitos sobre as responsabilidades municipais em saúde, sobre a rede de serviços de saúde que deveria ser criada a partir do PDR/RS, além da tabela de consultas e procedimentos em AB determinados pela NOAS-SUS.

A Portaria $n^{\circ}$ 1.101/01 do Gabinete M inisterial do M inistério da Saúde (PT. 1.101/01 - GM/ MS) foi utilizada nesteestudo para nortear a descrição e análise das ações em saúde executadas pelos municípios edispostas no DATASUS. A justificativa de sua utilização se deve por ser a portaria o documento legal existente para estabelecer parâmetros assistenciais para todos os níveis de atenção à saúde, da $A B$ à alta complexidade $(A C)$, e da capacidade instalada em leitos e equipamentos de saúde, relacionando sempreo quantitativo em estrutura e atendimento à saúde com a população do município. Porém, deve-se deixar claro que a referida portaria apresenta ape nas recomendações técnicas ideais, servindo de referência para orientar os gestores do SUS no planejamento, programação e priorização das ações de saúde a serem desenvolvidas; portanto, pode sofrer adequações regionais elocais deacordo com as diferentes realidades epidemiológicas efinanceiras ${ }^{9}$. 
Para fins de análise, as consultas da $A B$ foram descritas como consultas básicas urgentes (com remoção, sem remoção e com observação de até oito horas) e consultas básicas não urgentes (prénatal, clínica médica, ginecologia-obstetrícia, mastologia, pediatria, de Estratégia de Saúde da Família- ESF, domiciliar epara hanseníase). Relacionando os parâmetros ditados pela PT. 1.101/01 - GM/M S, a classificação do número absoluto de consultas informadas pelos municípios foi caracterizada de acordo com a sua adequação às normas propostas em: excedentes (acima detrês consultas por habitante ao ano - cons./hab./ano), adequadas (duas a três cons./hab./ano) e insuficientes (menos que duas cons./hab./ano).

Para a análise dos procedimentos básicos, foram definidos três grupos: procedimentos relacionados à saúde da mulher (coleta de material para exame citopatológico; consultas para indicação/fornecimento de diafragma; indicação/fornecimento/inserção deDIU), procedimentos clínicos ambulatoriais (testes específicos para diabetes mellitus - glicemia capilar, glicosúria e ce tonúria; eletrocardiograma; alta de tratamento específico supervisionado ou autoadministrado) e procedimentos cirúrgicos ambulatoriais (excisão e sutura simples; incisão e drenagem de abscesso; frenectomia; retirada de corpo estranho de cavidade nasal/auditiva).

Os dados referentes à capacidade instalada em serviços de saúde foram coletados do CNES/ DATASUS, de onde foram selecionados os serviços prestadores de atendimento na $A B$ em saúde caracterizados por postos de saúde (PS) e centros de saúde (CS). 0 CNES/DATASU Séum dos bancos de dados contidos no site do DATASUS e nele constam diversos dados referentes aos estabelecimentos de serviços de saúde existentes nos municípios brasileiros. Uma limitação dessebanco de dados é não fornecer um histórico dos estabelecimentos nele referidos, fazendo com que o dado seja considerado somente para o período em que foi coletado.

Além dos PS e CS, foram considerados os municípios que contam com atendimentos de ESF. Esta última classificação só foi possível por meio dos dados dispostos no SIA/DATASUS, de onde consta o item "consulta médica do PSF", que descreve a quantidade de consultas médicas realizadas por médico integrante de ESF; logo, não foram contadas unidades de saúde a mais quando identificados procedimentos pertencentes à ESF, somente acrescentou-se uma coluna a mais no quadro para mostrar a existência de tais consultas. Enfim, quanto à totalidade de estabe- lecimentos da $A B$ (soma de todos os estabelecimentos de $A B$ contidos no CNES/DATASUS), estes foram descritos como $A B$.

Os dados populacionais dos municípios foram organizados em tabela única no software Excel e utilizados para calcular os parâmetros assistenciais em consultas e para classificar o porte populacional dos municípios pesquisados. Para a classificação do porte populacional, realizou-se estratificação dos municípios estudados considerando a média populacional nos seis anos (2000-2005) pesquisados, a partir dos valores absolutos da população em cada município. Assim, os municípios foram classificados em: A municípios com população entre 50 e 70 mil habitantes, B - municípios com população entre 20 e 49.999 mil habitantes e $C$ - município com população menor que 20 mil habitantes.

Elaborou-se um perfil de utilização dos serviços de saúde de $A B$ para cada município, considerando a utilização efetiva (que relaciona oferta e demanda a valores absolutos de atendimentos prestados e registrados nos SIS pesquisados) e a capacidade instalada em serviços de saúde, através do cruzamento das variáveis utilizadas nos estudos.

Este projeto foi submetido e aprovado pelo Comitê de Ética em Pesquisa da Universidade Federal do Rio Grande do Sul, em 23 denovembro de 2006.

\section{Resultados}

Conforme o PDR/RS, a região em saúde do Rio Grande do Sul é composta de macrorregiões em saúde, com hospitais de referência macrorregional, que atendem aos casos mais complexos, ede microrregiões em saúde, com hospitais de referência microrregional eunidades locais de saúde (unidades mistas com atendimento ambulatorial e internações curtas de baixa complexidade). O PDR/RS 10 objetivou "sintetizar os principais conceitos e diretrizes que têm orientado a regionalização do sistema estadual, apresentar o diagnóstico da realidade de saúde do estado ea organizar a atenção e o sistema de referência proposto para o Estado do RS".

Com isso, o PDR/RS tentou criar caminhos capazes de suprir as demandas em saúde dos usuários/moradores da região em saúde do Rio Grande do Sul, propondo a consolidação decentros de referência e de especialidades, como uma redehierarquizada em que pequenos municípios se direcionem aos maiores e mais equipados (in- 
titulados “municípios-sede") para o atendimento integral à saúde.

A região em saúde que inclui os municípios estudados é composta por três macrorregiões Metropolitana, Sul e Vales, que se dividem em quatro microrregiões - Costa D oce Sul, Canguçu, São Lourenço do Sul e Cachoeira, e deve ter referências na assistênciaà saúde em seis municípios-sede - Camaquã, Dom Feliciano, Tapes, Canguçu, São Lourenço do Sul e Encruzilhada do Sul (Quadro 1).

Partiu-se dos conceitos e das divisões regionais firmados no PDR/RS, em que consta que os municípios nele citados apresentam capacidade instalada eficaz para atender suas próprias de mandas e, para os municípios-sede, também às demandas dos municípios pertencentes à mesma microrregião. M esmo o foco deste estudo sendo as ações da AB em saúde, que devem ser assumidas por todos os municípios brasileiros, os dados dos municípios foram organizados e analisados conforme o porte populacional e o PDR/RS, a partir dos seus municípios-sede (considerando estrutura em saúde e ações desenvolvidas).

A relação entre o porte populacional e a capacidade instalada em serviços de saúde do município evidenciou que o tamanho populacional está diretamenterelacionado à variedadeeà quantidade deestabelecimentos de AB. A pesar de não constar estabelecimentos de saúde em alguns municípios, conforme 0 item nível hierárquico do CNES/DATASUS, estes apresentaram algum tipo de unidade de saúde especificado, conforme o item relatório por unidade, também contido no CNES/DATASUS. Exemplo disto é o fato dos municípios de Sentinela do Sul e Chuvisca apresentarem, ao mesmo tempo, zero estabelecimento de saúde de $A B$ e um CS (Quadro 2).

Ambos os itens (nível hierárquico e relatório por unidade) são pertencentes ao CNES/DATASUS, o que torna essa informação contraditória epassível de ser interpretada como fal ha na apre sentação dos dados e/ou na metodologia empre gada, não permitindo clareza de dados. Além disso, não foi possível, conforme as fontes de dados utilizadas neste estudo, identificar quantas equipes de ESF existem em cada município. Essa informação não estava disposta no CNES/DATASUS e somente pôde ser identificada a partir do SIA/DATASUS, onde constavam consultas realizadas por profissionais médicos de PSF (Programa de Saúde da Família).

A quantidade de consultas básicas não urgentes apresentadas independe da estrutura em serviços de saúde disponível e, consequentemente, do tamanho populacional do município. Já quanto à diversidade destas consultas, os municípios de porte populacional C $(<20.000$ habitantes), com exceção de Arambaré, apresentaram predominantemente consultas em clínica médica sobre as demais. Santana da Boa Vista apresentou somente consultas em clínica médica em todo o período pesquisado (Quadro 3). Esses dados sugerem baixa diversidade de consul-

Quadro 1. Distribuição dos treze municípios em estudo conforme a regionalização da saúde.

\begin{tabular}{|c|c|c|c|}
\hline Macrorregião & Microrregião & Sede & M unicípio \\
\hline \multirow[t]{3}{*}{ M etropolitana } & Costa Doce Sul & Camaquã & $\begin{array}{l}\text { Amaral Ferrador } \\
\text { Arambaré } \\
\text { Camaquã } \\
\text { Cerro Grande do Sul } \\
\text { Chuvisca } \\
\text { Cristal } \\
\text { Sentinela do Sul }\end{array}$ \\
\hline & & Dom Feliciano & Dom Feliciano \\
\hline & & Tapes & Tapes \\
\hline \multirow[t]{2}{*}{ Sul } & Canguçu & Canguçu & $\begin{array}{l}\text { Canguçu } \\
\text { Santana da Boa Vista }\end{array}$ \\
\hline & São Lourenço do Sul & São Lourenço do Sul & São Lourenço do Sul \\
\hline Vales & Cachoeira & Encruzilhada do Sul & Encruzilhada do Sul \\
\hline
\end{tabular}

Fonte: Plano Diretor de Regionalização da Saúde, Rio Grande do Sul (2002). 
tas básicas não urgentes, associado a uma estrutura escassa em serviços de saúde nos municípios demenor porte, eevidenciam fal ha no processo de descentralização das ações e serviços da AB em saúde na região estudada.

Esses mesmos municípios, de porte populacional $\mathrm{C}$, apresentaram excesso de consultas não urgentes em diferentes períodos, sendo queChuvisca e A rambaré apresentaram esta situação em todo o período de estudo (Quadro 3). Quatro municípios do mesmo porte (Dom Feliciano, Tapes, Cerro Grande do Sul e Sentinela do Sul) apresentaram excessos na quantidade de consultas não urgentes em períodos variados. Este fato converge com a formulação (2002) e implantação (2003) do PDR/RS, sugerindo uma mudança inicial na organização das gestões municipais para 0 atendimento prestado, a partir da implantação de uma nova política estadual de saúde que visa à estruturação da rede de serviços de saúde municipal a partir das quatro áreas básicas - clínica médica, pediatria, ginecologia e obstetrícia e não somente a partir de pronto atendimentos ${ }^{8}$.

Municípios de maior portepopulacional (C), como Canguçu e São Lourenço do Sul, mostraram valores abaixo do esperado para a própria população (menos de duas cons./hab./ano) em consultas básicas não urgentes, conforme preconizado na PT 1.101 - GM/MS. Somente Cama- quã, dentre os municípios de porte populacional $C$, apresentou valores preconizados em quase todo o período (Quadros 2 e 3).

As quantidades de consultas básicas não urgentes adequadas (duas a três cons./hab./ano) foram apresentadas por nove dos treze municípios estudados, mesmo existindo diferenças no porte populacional e na estrutura em saúde desses municípios (Q uadro 3). Entretanto, a quantidade de consultas básicas não urgentes foi irre gular durante o período e nenhum dos municípios apresentou quantidades de consultas adequadas por mais de três anos seguidos, o que pode sugerir fragilidade na oferta, tanto por falta de profissionais competentes e comprometidos como por outros motivos.

Em relação às consultas básicas urgentes, observou-se que a maioria dos municípios apresentou déficit no período estudado. No entanto, entre os que notificaram tais consultas, predominaram as consultas básicas de urgência sem remoção. Somentecinco municípios, dediferentes portes populacionais (A - Camaquã, B - Encruzilhada do Sul eC - Amaral Ferrador, Cristal eDom Feliciano), apresentaram quantidades re comendadas de consultas (duasa três cons./hab./ ano). Dois deportepopulacional C (Amaral Ferrador e Cristal) apresentaram tais consultas em cinco dos seis anos pesquisados. Já Camaquã,

Quadro 2. Capacidade instalada em serviços de saúde de atenção básica e porte populacional nos treze municípios em estudo.

\begin{tabular}{|c|c|c|c|c|c|}
\hline \multirow[t]{2}{*}{ M unicípio } & \multirow[t]{2}{*}{$\begin{array}{c}\text { Porte } \\
\text { populacional }^{1}\end{array}$} & \multirow{2}{*}{$\begin{array}{c}\text { Nível } \\
\text { hierárquico² } \\
\text { AB }\end{array}$} & \multicolumn{2}{|c|}{$\begin{array}{c}\text { Relatório por } \\
\text { unidade }^{2}\end{array}$} & \multirow{2}{*}{$\begin{array}{l}\text { Consultas } \\
\text { médicas ESF } \\
\text { ESF }\end{array}$} \\
\hline & & & CS & PS & \\
\hline Camaquã & $A$ & 39 & 2 & 18 & $\mathrm{X}$ \\
\hline Canguçu & A & 15 & 5 & 13 & $x$ \\
\hline São Lourenço do Sul & B & 13 & 10 & 7 & $X$ \\
\hline Encruzilhada do Sul & $\mathrm{B}$ & 4 & 4 & 1 & $x$ \\
\hline Arambaré & B & 2 & - & 2 & $x$ \\
\hline Amaral Ferrador & $\mathrm{C}$ & 2 & 1 & 1 & $x$ \\
\hline Dom Feliciano & $\mathrm{C}$ & 3 & 3 & - & $x$ \\
\hline Tapes & $\mathrm{C}$ & 3 & 4 & - & $x$ \\
\hline Cerro Grande do Sul & C & 1 & 1 & - & - \\
\hline Sentinela do Sul & C & - & 1 & - & - \\
\hline Chuvisca & $\mathrm{C}$ & - & 1 & - & - \\
\hline Cristal & $\mathrm{C}$ & 2 & - & 2 & - \\
\hline Santana da Boa Vista & $\mathrm{C}$ & 5 & 1 & 3 & - \\
\hline
\end{tabular}

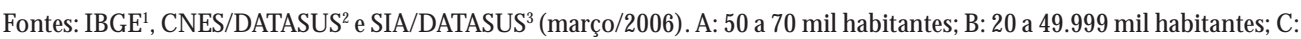
menos de 20 mil habitantes. AB: total de estabelecimentos de saúde de atenção básica; CS: total de centro de saúde; PS: total de posto de saúde; ESF: Estratégia deSaúde da Família; X: presença de consultas médicas de ESF notificadas no SIA/DATASUS. 
maior município de porte populacional pesquisado, apresentou déficit em consultas urgentes até 2002 , valores adequados nos dois anos seguintes e excesso em 2005. 0 excesso de consultas de urgência pode ser indicativo de fal has na oferta de serviços regulares, como as consultas básicas não urgentes e outras ações programáticas em saúde.

Municípios com porte populacional C (Cerro Grande do Sul, Chuvisca, Santana da Boa Vista, Sentinela do Sul e Tapes) e Canguçu (porte populacional A) não apresentaram consultas

Quadro 3. Adequação do número de consultas básicas não urgentes no período de 2000 a 2005 em relação aos parâmetros propostos na PT. 1.101 - GM/MS.

\begin{tabular}{|c|c|c|c|c|c|c|c|}
\hline Município & $A B^{2}$ & 2000 & 2001 & 2002 & 2003 & 2004 & 2005 \\
\hline Camaquã & 39 & & & & & & \\
\hline Canguçu & 15 & & & & & & \\
\hline São Lourenço do Sul & 13 & & & & & & \\
\hline Encruzilhada do Sul & 4 & & & & & & \\
\hline Arambaré & 2 & & & & & & \\
\hline Amaral Ferrador & 2 & & & & & & \\
\hline Dom Feliciano & 3 & & & & & & \\
\hline Tapes & 3 & & & & & & \\
\hline Cerro Grande do Sul & 1 & & & & & & \\
\hline Sentinela do Sul & 0 & & & & & & \\
\hline Chuvisca & 0 & & & & & & \\
\hline Cristal & 2 & & & & & & \\
\hline Santana da Boa Vista & 5 & & & & & & \\
\hline
\end{tabular}

Fontes: CNES/DATASU S² eSIA/DATASUS (março/2006). Legendas: AB: total de estabelecimentos deAB; célula branca (Déficit em consultas: <2 cons./hab./ano), célula cinza claro (Parâmetro recomendado: 2-3 cons./hab./ano), célula cinza escuro (Excesso em consultas: >3 cons./hab./ano).

Quadro 4. Adequação do número de consultas básicas de urgência no período de 2000 a 2005 em relação aos parâmetros propostos na PT. 1.101 - GM/MS.

\begin{tabular}{|c|c|c|c|c|c|c|c|}
\hline Município & $A B^{2}$ & 2000 & 2001 & 2002 & 2003 & 2004 & 2005 \\
\hline Camaquã & 39 & & & & & & \\
\hline Canguçu & 15 & & & & & & \\
\hline São Lourenço do Sul & 13 & & & & & & \\
\hline Encruzilhada do Sul & 4 & & & & & & \\
\hline Arambaré & 2 & & & & & & \\
\hline Amaral Ferrador & 2 & & & & & & \\
\hline Dom Feliciano & 3 & & & & & & \\
\hline Tapes & 3 & & & & & & \\
\hline Cerro Grande do Sul & 1 & & & & & & \\
\hline Sentinela do Sul & 0 & & & & & & \\
\hline Chuvisca & 0 & & & & & & \\
\hline Cristal & 2 & & & & & & \\
\hline Santana da Boa Vista & 5 & & & & & & \\
\hline
\end{tabular}

Fontes: CNES/DATASU S² eSI A/ DATASUS (março/2006). Legendas: AB: total de estabelecimentos deAB; célula branca (Déficit em consultas: $<2$ cons.hab./ano), célula cinza claro (Parâmetro recomendado: 2-3 cons.hab./ano), célula cinza escuro (Excesso em consultas: >3 cons.hab./ano). 
urgentes em todo o período estudado. São Lourenço do Sul (porte populacional $A$ ) não apresentou esse tipo de consultas até 2002 (Quadro 4). Essas informações podem revelar serviços municipais de saúde despreparados para atender às demandas espontâneas da população, ou mesmo o desvio desse tipo de atendimento para serviços de MC.

Os municípios que apresentaram maiores quantidades de consultas básicas urgentes podem ter recebido usuários demunicípios vizinhos para tais atendimentos. Supõe-se tal assertiva com dados referentes aos municípios de Amaral Ferrador (6,13 cons./hab./ano em 2002) e Cristal ( 6,45 cons./hab./ano em 2004), que apresentaram valores acima de duas vezes o recomendado para sua população, e com dados de EncruziIhada do Sul, que chegaram a quase três vezes a quantidade máxima recomendada em 2001 (8,62 cons./hab./ano). Além disso, esses mesmos municípios também notificaram consultas não urgentes em quantidades adequadas para os mesmos anos descritos, o que descarta a possibilidade de seus usuários acessarem os serviços de $A B$ somente em casos de urgência.

A quantidade e variedade de procedimentos básicos dependem da estrutura em saúde dos municípios. Cristal não seguea mesma regra, pois, mesmo sendo um município de porte populacional C (Quadro 2), apresentou procedimentos variados (em saúde da mulher, clínicos ambulatoriais e cirúrgicos ambulatoriais) em todo o pe- ríodo estudado (Quadro 5). Dos cinco municípios com CS, PS e ESF, somente São Lourenço do Sul apresentou os três grupos de procedimentos em todo o período de estudo (Quadro 5).

Procedimentos cirúrgicos e ambulatoriais predominaram entre todos os procedimentos básicos apresentados, evidenciando o caráter de urgência nas ações queenvolvem procedimentos ofertados na $A B$ em saúde. Esses procedimentos envolvem, entreoutros, excisões, incisões, sutura simples e retirada de corpo estranho da cavidade nasal e/ou auditiva. Arambaré até 2004, Cerro Grande do Sul até 2001, Amaral Ferrador até 2001, Chuvisca em 2002 e Tapes em 2005 apresentaram apenas esses tipos de procedimentos.

Os dados apresentados indicam um fluxo de usuários entre municípios para a satisfação de necessidades em procedimentos básicos. Logo, os usuários podem ter se direcionado dos municípios que apresentaram maior ausência de procedimentos básicos (Arambaré, Tapes, Cerro Grande do Sul, Chuvisca, Sentinela do Sul eSantana da Boa vista) para outros municípios da região que disponibilizaram tais procedimentos.

\section{Discussão}

Sabe-se que a análise da utilização dos serviços de saúde por meio de valores absolutos de consultas e procedimentos realizados não permite a descrição de dinâmicas locais, porém pode auxi-

Quadro 5. Distribuição do agrupamento dos procedimentos básicos notificados no SIA/DATASUS pelos treze municípios em estudo no período de 2000 a 2005.

\begin{tabular}{|c|c|c|c|c|c|c|c|c|c|c|c|c|c|c|c|c|c|c|}
\hline \multirow{2}{*}{ Município } & \multicolumn{3}{|c|}{2000} & \multicolumn{3}{|c|}{2001} & \multicolumn{3}{|c|}{2002} & \multicolumn{3}{|c|}{2003} & \multicolumn{3}{|c|}{2004} & \multicolumn{3}{|c|}{2005} \\
\hline & I & II & III & I & II & III & I & II & III & I & II & III & I & II & III & I & II & III \\
\hline Canguçu & $x$ & $x$ & & $x$ & $x$ & $x$ & $x$ & $x$ & $x$ & $x$ & $x$ & $x$ & $x$ & $x$ & $x$ & $x$ & $x$ & $x$ \\
\hline São Lourenço do Sul & $x$ & $x$ & $x$ & $x$ & $x$ & $x$ & $x$ & $x$ & $x$ & $x$ & $x$ & $x$ & $x$ & $\mathrm{x}$ & $x$ & $x$ & $x$ & $x$ \\
\hline Camaquã & & $x$ & $x$ & $x$ & $x$ & $x$ & $x$ & $x$ & $x$ & $\mathrm{x}$ & $x$ & $\mathrm{x}$ & $x$ & $x$ & $x$ & $x$ & $x$ & \\
\hline Encruzilhada do Sul & $x$ & $x$ & $\mathrm{x}$ & $x$ & $x$ & $x$ & $x$ & & $x$ & & $x$ & $x$ & $x$ & $\mathrm{x}$ & $x$ & $x$ & $x$ & $x$ \\
\hline Amaral Ferrador & & & $x$ & & & $\mathrm{x}$ & $x$ & & $x$ & $\mathrm{x}$ & & $x$ & $x$ & & $x$ & $\mathrm{x}$ & $\mathrm{x}$ & $\mathrm{x}$ \\
\hline Dom Feliciano & $x$ & $x$ & $x$ & $x$ & $\mathrm{x}$ & $x$ & $x$ & $x$ & $x$ & $\mathrm{x}$ & $\mathrm{x}$ & $\mathrm{x}$ & $x$ & $x$ & $x$ & $\mathrm{x}$ & $x$ & $x$ \\
\hline Arambaré & & & $\mathrm{X}$ & & & $\mathrm{X}$ & & & $\mathrm{X}$ & & & $\mathrm{X}$ & & & $\mathrm{X}$ & $\mathrm{X}$ & $\mathrm{X}$ & $\mathrm{X}$ \\
\hline Tapes & & & $x$ & & & $\mathrm{x}$ & & & $x$ & & & & $\mathrm{x}$ & $x$ & & & & $x$ \\
\hline Cerro Grande do Sul & & & $x$ & & & $x$ & $x$ & & $x$ & $\mathrm{x}$ & & $x$ & & & & & & \\
\hline Chuvisca & & & & $x$ & & & & & $x$ & $x$ & & $x$ & $\mathrm{x}$ & $x$ & & $x$ & $x$ & $x$ \\
\hline Sentinela do Sul & & & & & & & $x$ & & & & & & & & & & & \\
\hline Cristal & $x$ & & $\mathrm{x}$ & $\mathrm{x}$ & $\mathrm{x}$ & $\mathrm{x}$ & $x$ & $\mathrm{x}$ & $\mathrm{x}$ & $\mathrm{x}$ & $x$ & $x$ & $\mathrm{x}$ & $x$ & $x$ & $\mathrm{x}$ & $x$ & $x$ \\
\hline Santana da Boa Vista & & & & & & & & & & & & & $x$ & & & $x$ & $x$ & \\
\hline
\end{tabular}

Fontes: SI A/DATASUS (março/2006). Legenda: I - Procedimentos relacionados à saúde da mulher; II - Procedimentos clínicos ambulatoriais; III - Procedimentos cirúrgicos ambulatoriais. 0 "x" refere-seà presença dos procedimentos notificados no SIA/DATASUS. 
liar na identificação de possíveis disparidades re gionais e funcionais relacionadas à oferta eà demanda por serviços de saúde. Pode, ainda, fornecer pistas de iniquidades existentes, pois a utilização dos serviços desaúde éum processo complexo que resulta de um conjunto amplo de determinantes, abrangendo desde fatores de organização da oferta dos serviços até as características demográficas, econômicas, sociais eculturais dos usuários ${ }^{11}$.

Os resultados encontrados mostraram uma diversidade de situações na $A B$ em saúde para a região pesquisada, desde a ausência (ou mesmo o excesso) de atendimentos básicos em períodos variados para alguns municípios, até 0 atendimento constante em todo o período de 2000 a 2005. Este estudo utilizou dados secundários como base de análise, por isso os resultados possibilitaram diferentes interpretações para o fenômeno encontrado. Pode-se citar a existência de um banco de dados falho, com subnotificações das consultas e procedimentos realizados na $A B$ em saúde, ou mesmo uma ausência de oferta e/ou demanda por serviços de $A B$ nos municípios estudados.

Para a hipótese de subnotificações dos bancos de dados, pode-se falar em baixa capacitação dos profissionais que lidam com os SIS localmente, ou mesmo a rotatividade defuncionários nessa função, o que faz com que não consigam se adaptar ao sistema de informações e não entendam os seus objetivos posteriores de uso, como o de planejar as ações em saúde que resultem na melhoria da qualidade de vida da sua população.

No dia a dia dos serviços de saúde, observase os profissionais coletando um grande número de dados, preenchendo formulários e mais formulários, os encaminhando às secretarias municipais de saúde e não obtendo retorno das informações que ajudaram a gerar. Percebe-sequeeste descaso com os profissionais "da ponta" pode desmotivá-los na execução da alimentação dos SIS e, ao mesmo tempo, perde-se a oportunidade de promover uma discussão a respeito da importância de sealimentar corretamente os SIS, pois nem todo o dado que é coletado se transforma em informação ${ }^{6}$.

Ao insinuar a existência de um banco de dados fal ho, pensa-se nas modificações metodológicas dos SIS, com alterações técnicas nos seus programas e sem os devidos cuidados prévios, 0 que poderesultar em inacessibilidadee perda dos dados contidos. Exemplo de discordância de dados em um único banco, como já exposto nos resultados deste estudo, está no que refere à estrutura do CNES/DATASUS, que indica quantidades diferentes de unidades de saúde na $A B$ em um mesmo município, dependendo somente de como é apresentada a classificação utilizada.

N este exemplo, o item nível de hierarquia refere-se aos diferentes serviços de AB, MC eAC, e 0 item relatório por unidade apresenta as diferentes unidades de serviço, como os CS e os PS. Desta maneira, o SIS permitequesuas partes ope rem de maneira desconexas, gerando informações incoerentes em um mesmo banco de dados.

A implantação dos sistemas locais de informação em saúde possi bilitaria a organização dos municípios, que poderiam "subsidiar decisões administrativas, como priorização de recursos, localização e dimensionamento de unidades de saúde, estabelecimento de perfis profissionais dos recursos humanos e dos treinamentos e atualizações necessárias" ${ }^{12}$. Esta estratégia, se implantada em consonância com os níveis estadual e federal, pode contribuir para a qualificação dos SIS no sentido de que estes tenham uma maior responsabilidade sobre os dados coletados e as informações geradas.

Considerando uma ausência de oferta e/ou de demanda por serviços de $A B$ nos municípios citados, pode-se sugerir a existência de fluxos intermunicipais para suprir necessidades em saúde na $A B$, justificando, assim, os excessos e os déficits em serviços de $A B$ em alguns municípios. É sabido que esses fluxos não são preconizados pe los mecanismos reguladores da assistência aqui apresentados (NOAS-SUS, PT. 1.101 - GM /M S, PDR/RS); porém, esses mecanismos parecem ter interferido pouco na quantidade ediversidade de consultas e procedimentos apresentados pelos municípios quanto à municipalização da $A B$.

Outras questões podem ser pensadas a partir do exposto no estudo, como a falta de oferta de serviços na $A B$ que pode ter gerado um fluxo de usuários, primariamente da $A B$, para os serviços de MC. Além disso, falhas no processo de efetivação da regional ização edescentralização dos serviços básicos de saúde também podem acarretar uma concentração dos serviços de $A B$ em apenas alguns municípios, ou mesmo a escassez em estrutura e oferta destes serviços nos municípios de menor porte.

Em estudo epidemiológico desenvolvido no município de Pelotas, observaram-se alguns fatores relacionados às consultas médicas acima da média: condições socioeconômicas, sexo, idade, presença de doenças crônicas e ocorrências de pessoas que foram hospitalizadas no último 
ano. Os resultados deste estudo apontam que 0 elevado número de consultas pode refletir a necessidade de qualificação profissional ${ }^{13}$. Estes re sultados são semelhantes aos de outro estudo realizado no mesmo município ${ }^{14}$.

O utra questão extremamenterelevanteéa de quemuitosformulários são preenchidos em função da produtividade e, em razão disso, podem ocorrer algumas distorções. Em trabalho desenvolvido sobre a informação e a decisão política em saúde, émencionado queas informações produzidas pelas grandes bancos de dados possuem, predominantemente, uma dimensão financeira ${ }^{15}$. Esse fato possibilita o questionamento sobre possíveis alterações nos dados em alguns municípios em virtude do financiamento, que ocorre em função das informações contidas nesses sistemas; porém, o estudo a partir de dados secundários não responde a estas questões.

Sabe-se queénecessário aprofundar a análise da oferta e demanda de saúde a partir da realidadelocal epossibilitar o desvelamento depossíveis disparidades locais que possam ocorrer, através do delineamento dos possíveis fluxos, trajetórias e itinerários terapêuticos dos usuários da $A B$ em saúde. Porém, entende-se também que a visualização dessas possíveis disparidades e desigualdades na utilização dos serviços deveria estar mais explicitada, para que o reconhecimento dos pontos a serem trabalhados na melhoria do acesso aos serviços de saúde e o planejamento em saúde nos municípios fossem realmente efetivos.

Em pesquisa recenterealizada com municípios de pequeno porte, Cecílio et al. ${ }^{16}$ descreveram que os gestores de saúde municipais pouco conhecem da sua realidade local, mostrando o seu despreparo e, ao mesmo tempo, a importância de estudos e programas que desenvolvam a capacidade dos gestores para planejar com base nos fatos. Nesta busca por resultados positivos que impactem a saúde da população nos municípios, os dados provenientes dos SIS, independentemente se tratados no âmbito local ou não, mostram-se importantes ferramentas para guiar as ações dos gestores locais de saúde.

N ormalmente, o foco de atenção dos gestores municipais se concentra no desenvolvimento de programas tradicionais dos governos federal e estadual, quando poderiam trabalhar na mudança e inovação das políticas de saúde ${ }^{15}$. Isso revela o despreparo e a falta de articulação das gestões em saúde para desenvolver, de forma concomitante, suas políticas locais e as políticas instituídas pelas outras esferas governamentais que têm de ser cumpridas localmente.

\section{Considerações finais}

Os resultados deste estudo, evidenciados por meio da análise de dados secundários, apontam disparidades entre os diferentes municípios, mostrando que alguns deles não estão adequados à PT. 1101 - GM /M S eà N OAS-SUS. Considerando as variáveis pesquisadas (procedimentos e consultas realizados, estrutura populacional e organização da rede de serviços de AB), estas nos levam a pensar na existência de uma de sigualdade "regional" de acesso à $A B$ em saúde entre os municípios estudados.

Alguns problemas em relação aos grandes bancos de dados dos SIS devem estar ocorrendo, como as subnotificações e as falhas metodológicas, além dos problemas locais, tais como a falta de oferta dos serviços de $A B$, utilização de serviços de $M C$ para ações sensíveis à $A B$ e problemas no preenchimento dos formulários devido a questões relacionadas à produtividade. Parece que a qualidade da informação, sobretudo em municípios de pequeno porte, revela, na realidade, a qualidade da alimentação dos dados em todas as etapas do processo até a disponibilização pública dos dados nos SIS.

Acredita-se que o estudo proporcionou o le vantamento de algumas questões importantes; porém, não houve a pretensão de esgotar 0 assunto, sendo necessários outros estudos que problematizem este tema, visto a escassa bibliografia referente às dificuldades estruturais dos SIS, especial mente na pesquisa de dados secundários e na formulação de políticas públicas em saúde.

Os resultados apresentados apontaram para uma questão essencial que se refere à qualidade da informação em saúde, que compromete o uso da informação no processo de planejamento de ações em saúde. Embora as razões sejam oriundas de diferentes motivos, fica evidente, como fora apontado também por M oraes e Santos ${ }^{17}$, que o processo de gestão em saúde ainda se dá sob uma lógica fragmentadora evertical, pouco sintonizada com a diversidade que caracteriza a situação de saúde da população brasileira, acarretando uma resistência ao uso na gestão da saúde de tecnologias integradoras e flexíveis que instrumentalizam uma abordagem mais globalizante e próxima da realidade sanitária, como propiciam as novas tecnologias em saúde.

A qualidade do cuidado em saúde pode ser aprimorada se as decisões profissionais forem baseadas em informações atualizadas e confiáveis. Um dos grandes desafios para o profissional da saúde e para os gestores locais é manejar 0 
volume crescente de informações na busca dos dados mais relevantes, que auxiliarão na visualização de problemas e da utilização dos serviços de saúde pela população. Os SIS podem ser importantesaliados, desde que, diante de um excesso de informações presentes no cotidiano dos serviços, os profissionais sejam capazes de ter acesso, responsabilidade e compromisso de transformar a informação em ação. Esteéum desafio que necessita fazer parte da Agenda Política da Saúdee fomentar um processo de construção de SIS que não sejam pensados como uma "caixa preta" para os gestores, utilizando-se da expressão de M oraes e Gómez ${ }^{18}$. Ao contrário, que sejam apreendidos como imprescindíveis para a constituição do campo do planejamento em saúde.

\section{Colaboradores}

TE Gerhardt foi responsável pela coordenação, orientação e acompanhamento da pesquisa e a elaboração do artigo; JM Pinto e DL Riquinho realizaram a coleta, descrição, interpretação eanálise dos dados e elaboração deste artigo; A Roese auxiliou na interpretação eanálise dos dados ena redação deste artigo e DL Santos e M C Lima auxiliaram na coleta dos dados de pesquisa. 


\section{Referências}

1. Universidade Federal do Rio Grande do Sul. Escola de Enfermagem. Programa de Pós-Graduação em Enfermagem. Fluxos e utilização de serviços de saúde: mobilidade e necessidades em saúde de usuários e novos desafios para a integralidade em saúde pública [relatório de pesquisa]. Porto Alegre: Universidade Federal do Rio Grande do Sul; 2008.

2. Mattos RA. Repensando a organização da rede de serviços de saúde a partir do princípio da integralidade. In: Pinheiro R, M attos RA, organizadores. Razões públicas para a integralidade em saúde: 0 cuidado como valor. Rio de Janeiro: CEPESC-UERJ/ ABRASC0; 2007. p. 369-383.

3. Brasil. Lei n- 8.080, de 19 de Setembro de 1990. Dispõe sobre as condições para a promoção, proteção e recuperação da saúde, a organização e o funcionamento dos serviços correspondentes e dá outras providências. Diário O ficial da União 1990; 19 set.

4. Sawyer DO, Leite IC, Alexandrino R. Perfis de utilização de serviços de saúde no Brasil. Cien Saude Colet 2002; 7(4):757-776.

5. Cecilio LCO. As necessidades de saúde como conceito estruturante na luta pela integralidade e equidade na atenção em saúde. In: Pinheiro $R, M$ attos RA, organizadores. Os sentidos da integralidade na atenção e no cuidado à saúde. Rio de Janeiro: IMSUERJ/BRASCO; 2001. p.113-126.

6. Mota E, Carvalho DM. Sistemas de Informação em Saúde. In: Rouquayrol MZ, Almeida Filho N, organizadores. Epidemiologia e Saúde. 6a ed. Rio de Janeiro: Medsi; 2003. p. 605-628.

7. Finkelman J. Caminhos da saúde pública no Brasil. Rio de Janeiro: Fiocruz; 2002.

8. Brasil. Resolução n 373, de 27 de fevereiro de 2002. Dispõe sobre a Norma Operacional da Assistência à Saúde. Diário Oficial da União 2002; 28 fev.

9. Brasil. Portaria n 1.101, de 12 de junho de 2002. Dispõe sobre os parâmetros de cobertura assistencial no âmbito do Sistema Único de Saúde - SUS. Diário Oficial da União 2002; 13 jun.
10. Rio Grande do Sul. Secretaria de Estado da Saúde. Plano Diretor de Regionalização da Saúde. Rio Grande do Sul: Secretaria de Estado da Saúde; 2002.

11. Santos DL. Determinantes sociais, acesso e utilização de serviços de saúde no município de Camaquã/RS [projeto de mestrado]. Porto Alegre: Escola de Enfermagem da UFRGS; 2008.

12. Pinho VP. Sistema de informações para a gestão local de saúde. Saúde e Sociedade 1995; 4(1⁄2):47-50.

13. Dias-da-Costa JS, Olinto MTA, Gigante DP, Menezes AM B, M acedo S, Daltoé T, Santos I S, Fuchs SC. Utilização de serviços ambulatoriais de saúde em Pelotas, Rio Grande do Sul, Brasil: alguns fatores relacionados com as consultas médicas acima da média. Cad Saude Publica 2008; 24(2):353-363.

14. Capilheira M F, Santos IS. Fatores individuais associados à utilização de consultas médicas por adultos. Rev. Saude Publica 2006; 40(3):436-443.

15. Cohn A, Westphal MF, Elias PE. Informação e decisão política em saúde. Rev. Saude Publica 2005; 39(1):114-121.

16. Cecílio LCO, Andreazza R, Souza ALM, Lima MR, M ercadante CEB, Pinto NRS, Spedo SM, Sato WNS, M arcadante CEB, Veja C, Bestetti LM A, Lima M R. O gestor municipal na atual etapa de implantação do SUS: características e desafios. R. Eletr. de Com. Inf. Inov. Saúde 2007; 1(2):200-207.

17. M oraes IHS, Santos SRR. Informações para a gestão do SUS: necessidades e perspectivas. Inf Epidemiol SUS 2001; 10(1):49-56.

18. Moraes IHS, Gómez M NG. Informação e informática em saúde: caleidoscópio contemporâneo da saúde. Cien Saude Colet 2007; 12(3):553-565.

Artigo apresentado em 01/11/2008

Aprovado em 05/06/2009

Versão final apresentada em 19/07/2009 